Women who have sex with women (WSW) were reluctant to attend services due to perceptions of low risk and discrimination, and valued the choice of a women-only service).

In 2012 a women's clinic opened, offering a range of sexual health and contraception services. Staffed by female HCPs and receptionists, the service has been well received by women. Plans for a women-only waiting area proved challenging within the confines of environment and patient activity.

Aim(s)/objectives To assess patient experience of the women's clinic, including that of mixed sex versus female only waiting areas.

Methods An anonymous patient experience questionnaire distributed $3^{\text {rd }}-17^{\text {th }}$ April 2014 . Women were asked their age, sexual orientation, previous experience of services and their views on accessing integrated contraception and sexual health care. Data was collated and entered into an excel database.

Results Questionnaires were received from 43 women (36 fully completed); Majority $(\mathrm{n}=21,50 \%) 26-35$ years. $33(77 \%)$ WSM, 3 (7\%) WSW; 7 (16\%) did not answer. 28 (66\%) had accessed other sexual health/ contraception services within 3 years. $3(6 \%)$ preferred female only waiting areas, with 40 (94\%) wanting a choice, or stating that they had no strong feelings.

Discussion Assumptions about acceptability of single-sex waiting areas did not match the majority of patients' views. WSM and WSW accessing the service valued the choice of mixed or single sex waiting areas.

\section{P250 SEXUAL HEALTH INFORMATION AND SERVICES: THE VIEWS AND EXPERIENCES OF 14 TO 22 YEAR OLDS}

${ }^{1}$ Babs Evans*, ${ }^{2}$ David Armitage. ${ }^{1}$ Nudge Associates, London, UK; ${ }^{2}$ Tameside Metropolitan Borough Council, Ashton-Under-Lyne, UK

\subsection{6/sextrans-2015-052126.292}

Background/introduction Young people are not always consulted about their sexual health information and service needs.

Aim(s)/objectives The authors sought to capture young people's views and experiences of sexual health information and services in a specific geographical area.

Methods An online survey was published on survey monkey between 4 and 16 December 2014. It was promoted via social media, youth groups and Lesbian, Gay, Bisexual and Transgender (LGBT) organisations. 207 responses from young people aged between 14 and 22 were analysed.

Results $50 \%$ of respondents were female. Of 190 stating sexuality, $12 \%$ may be gay or bisexual. Only $13 \%$ had attended sexual health classes that met all their sexual health needs. Young people reported getting sexual health information from TV programmes and websites. Young women were more likely to get information from family members than young men. Most young people knew where they could get condoms, pregnancy tests and emergency contraception. 85\% did not know about PEP (Post Exposure Phrophylaxis) for HIV. 30 young women had talked to a health professional about contraception, most commonly the pill and implant. Young people want sexual health services to be open in the evenings and weekends, the most common combination was Monday evening, Friday evening, and Saturday afternoon.

Discussion/conclusion The sexual health information needs of young people are not being met in education settings. More information about PEP is needed, especially for young gay and bisexual men. Sexual health services should have extended opening hours leading up to, during and after weekends.

\section{P251 TREATMENT DILEMMA OF CHLAMYDIA IN PREGNANCY}

Jemy Thomas*, CM Bates, T Mathew. Royal Liverpool University Hospital, Liverpool, UK

\subsection{6/sextrans-2015-052126.293}

Background Drug hypersensitivity reactions are immunological responses to medications. An accurate understanding of the type of antibiotic hypersensitivity reactions is crucial in the decision making process of alternative antibiotic usage versus desensitisation.

Clinical presentation A 25-year old female, twenty-four weeks pregnant, with dysuria was diagnosed with Chlamydia. She had asthma, which was treated with inhalers. She gave a history of reaction to penicillin and an episode of collapse and rash to erythromycin. Effective treatments for Chlamydia are azithromycin, erythromycin, amoxicillin and doxycycline. The latter is contraindicated in pregnancy and erythromycin and amoxicillin were contraindicated because of this patient's history. There is small risk of cross reactivity between azithromycin and erythromycin, so a desensitisation protocol was drawn up by the immunologist. The patient was counselled regarding the possibility of a reaction even to small doses of azithromycin and the possibility of an anaphylactic reaction needing adrenaline, which could precipitate preterm labour. She was admitted on the ward and given azithromycin in titrating doses, which was tolerated well without any problems. The repeat chlamydia test following treatment was negative.

Discussion There are limited therapeutic choices for treatment of various sexually transmitted infections in patients with allergies particularly in pregnancy. These patients will need desensitisation under an immunologist with careful monitoring. If a patient with a reported allergy is deemed not allergic or if the allergy is simply an expected side effect, the medical record should be updated to reflect this change along with educating the patient.

\section{P252 TILL DEATH DO US PART: MARRIAGE, AFRICAN-BORN WOMEN AND HIV PREVENTIATION IN THE UNITED KINGDOM}

Tabeth Timba-Emmanuel*, Thilo Kroll, Mary Renfrew. University of Dundee, Dundee, UK

\subsection{6/sextrans-2015-052126.294}

Background/introduction Recent studies from Sub-Saharan Africa, most especially Southern Africa, reveal a shocking trend in HIV transmission with married couples recording the biggest percentage of new infections per annum. Hence the mode of transmission as far as HIV is concerned has been evolving and the previously so called 'low risk' unions are no longer as safe as previously thought, most especially for women. UK literature shows that the trend of HIV in Black-African population mirrors that in Africa. Making of culturally sensitive and therefore effective policies and interventions for this particular group calls for a good in-depth understanding and insight into experiences and strategies that persists and those that newly emerge for married African-born women when they immigrate into UK. 